\title{
YKL-40 - A NEW DIAGNOSTIC BIOMARKER FOR BENIGN BREAST DISEASES AND BREAST CANCER
}

\author{
V. Uzunova ${ }^{1}$, G. Paskalev ${ }^{1}$, M. Kazakova ${ }^{3}$, E. Poryazova² ${ }^{2}$ K. Murdjev ${ }^{1}$, A. Uchikov ${ }^{1}$, \\ A. Iankulov ${ }^{1}$, V. Sarafian ${ }^{3}$, D. Manolova ${ }^{5}$, A. Paskaleva ${ }^{4}$, B. Hadjiev ${ }^{1}$, J. Grudeva- \\ Popova $^{6}$ \\ 1) Clinic of Thoracic and Abdominal Surgery, 2) Department of Common and \\ Clinical Pathology, 3) Department of Biology, 4) Clinic of Dermatologic and \\ Veneric Diseases, 5) Department of Diagnostic Imaging, 6) Department of \\ Oncology and Haematology \\ Medical University - Plovdiv, Bulgaria
}

\begin{abstract}
:
Benign breast diseases encompass a wide spectrum of lesions, which raise a lot of questions about their classification, diagnosis, prognosis and surgical treatment.

The aim of this study is to measure the serum level of YKL-40 in cases of different groups of benign breast diseases, to compare it to the level of healthy women as well as to those with breast cancer and to examine its tissue expression after surgical treatment. We use it as a diagnostic marker and as a criterion for differential diagnosis.

Forty nine (49) patients with benign breast diseases and twenty (20) patients with breast cancer were examined. All of them had their serum level of YKL-40 measured preoperatively and its tissue expression examined immunohystochemically after the surgical intervention. There were significant differences in both concentration and tissue expression of this marker in patients with different groups of benign breast diseases and breast cancer. YKL-40 can be an important biomarker in the diagnosis and differential diagnosis of breast diseases.
\end{abstract}

Key words: breast, YKL-40, benign diseases, biomarker

\section{INTRODUCTION}

YKL-40 is a new biomarker, which represents a heparin-binding and chitin- binding glycoprotein. It belongs to a group of mammalian proteins with an amino acid sequence similar to that of 18 glucosyl hydrolases, a group of bacterial chitinases (1), although YKL-40 does not have a chitinase activity. It is possible that YKL-40 has a role in the process of angiogenesis, stimulating the endothelial cells, and contributes to the formation of branching tubules. It is also well established that YKL-40 is a potent growth factor inducing the proliferation of chondrocytes and fibroblasts. YKL-40 links to the starting mechanism of reticuloendothelial system (RES) $(2,3)$. The characteristics of human YKL-40 gene
(CHI3L1) as well as the crystal structure of the protein are already known, but a cell receptor for YKL-40 has not yet been identified. Its physiological action is not well studied. The biological function of YKL-40 is not known $(4,5)$. It is supposed that YKL-40 maybe has a role in the proliferation and differentiation of malignant cells; maybe it protects tumor cells from apoptosis, stimulates angiogenesis; participates in the extracellular tissue remodeling; stimulates fibroblasts around the tumor; but this hypothesis still has to be confirmed in vivo (6). Up to now there are no studies on the expression of YKL-40, or on its serum level, in benign breast lesions. There is no data about the possibility of using YKL-40 as an early marker for the transformation of benign lesions into malignant ones, nor there is data in the literature about the association between its serum levels and its immunohystochemical expression in benign breast lesions and breast cancer.

THE AIM of this study is to measure preoperatively the serum level of YKL-40 in patients with benign breast diseases, and to compare it to its tissue expression, so that this may help in the diagnosis and differential diagnosis of breast cancer.

\section{MATERIALS AND METHODS}

Thirty nine /39/ women with benign breast diseases (including 6 with intraductal papilloma; 13 with fibroadenoma; 5 with cysts; 1 with phyllodes tumor; 10 with fibrocystic changes; 1 with lipoma and 3 with atypical ductal hyperplasia) and twelve /12/ women with breast cancer (11 of which with invasive ductal carcinoma and 1 with lobular carcinoma) were examined, diagnosed and underwent surgical treatment. The serum level of YKL-40 of ten /10/ healthy controls was examined as well - these were young women (aged 20-31 years) without family history of breast or ovarian cancer, who 
were not on hormonal therapy, with no clinical or ultrasound signs of breast diseases or inflammatory diseases.

The examined women were between 18 and 73 years of age. On the basis of clinical, ultrasound and mammographical signs they were diagnosed as follows: fibroadenoma (14 women), intraductal papilloma (6 women), cysts ( 7 women), phyllodes tumor (1 woman), fibrocystic changes (10 women), and breast cancer (12 women). All women underwent surgical treatment. Sectoral excision was performed in 28 women, microdochectomy in 6 , wide excision in 5, quadrantectomy in 2 and mastectomy with lymph node dissection in 10 . The diagnosis was confirmed hystologically in all patients: intraductal papilloma in 6 , cysts in 5, atypical ductal hyperplasia in 3, phyllodes tumor in 1, fibrocystic changes in 10 and fibroadenoma in 12. In one of the patients with a preoperative diagnosis of fibroadenoma, the tumor turned out to be a breast cancer intraoperatively, which necessitated the change to quadrantectomy with lymph node dissection. The serum level of YKL-40 was examined preoperatively in all women. An enzyme-binding immunosorbent test (ELISA) was applied according to the producer's instructions. A specific kit Microvue YKL-40 EIA

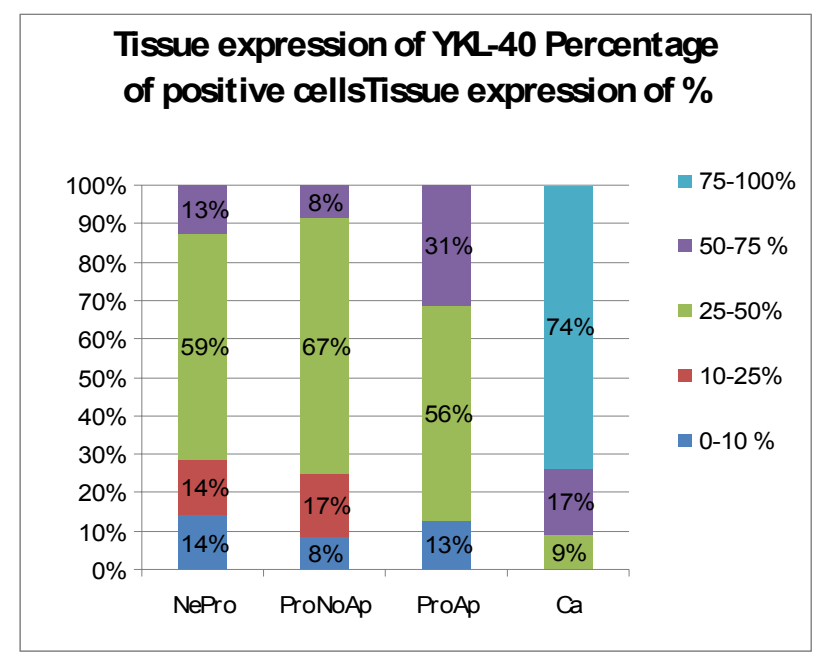

\section{DISCUSSION}

Different groups of authors indicate that the serum level of YKL-40 in healthy individuals is 42 micrograms (1, 9 ). In our control group of healthy women (without family history of breast or ovarian cancer, without hormonal or contraceptive therapy, non smokers, without inflammatory diseases or diseases of liver and lungs) the mean serum level was $41,11 \mathrm{ng} / \mathrm{ml}$; the level slightly, but insignificantly, increased with age. Our study showed that the serum level of YKL-40 in nonproliferating benign breast diseases was near that of healthy women. The level increased in patients with proliferating diseases without atypia as well as in diseases with atypia. The level of YKL-40 in patients with breast cancer was significantly higher in comparison with patients with nonproliferating benign breast diseases (table
Kit was used.

After the surgical procedure the percentage of cells positive for YKL-40 was determined immunohystochemically as well as the intensity of staining. Clear cytoplasmic staining was established as a positive sign. Two pathologists examined each preparation twice. The mean value of staining intensity and percentage of positive cells was accepted as definitive.

\section{RESULTS}

The preoperative serum level of YKL-40 in women with benign breast diseases was as follows: $44,58 \mathrm{ng} / \mathrm{ml}$ for the group of nonproliferating lesions (fibroadenoma, cysts, fibrocystic changes without proliferation); $55,5 \mathrm{ng} / \mathrm{ml}$ for the group of proliferating lesions without atypia; $78,4 \mathrm{ng} / \mathrm{ml}$ for the group of proliferating lesions with atypia; and 100,27 ng/ $\mathrm{ml}$ for the cancer group.

The immunohystochemical examination of YKL-40 in the tissues of the different groups of benign breast diseases is shown on fig.1. For the breast cancer cells the tissue expression is in the range of $75-100 \%$ and the staining intensity is moderate to strong (fig.2).

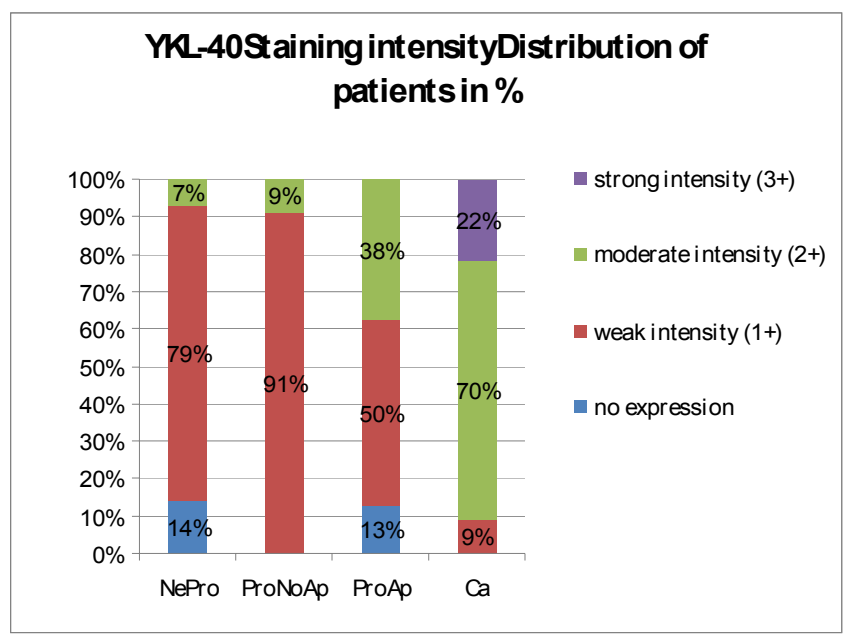

1). According to Johansen and co.(11, 13) serum concentration of YKL-40 above $20 \%$ is indicative of increased serum level. In our patients changes in levels were above $25 \%$. These results show that the increasing of serum concentration of YKL-40 can be a sign of increasing risk of breast cancer development.

Some authors $(1,12)$ consider that the tissue expression in breast cancer is positive. Our study demonstrated that tissue expression in the group of nonproliferating benign breast diseases (fibroadenoma, cysts without atypia) was weak as was the intensity of staining; in the group of proliferating diseases (papilloma, sclerosing adenosis) the percentage of positive cells, expressing the marker, was $25-50 \%$ and the intensity of staining was moderate. In breast cancer the percentage of cells, expressing 
YKL-40, was $75-100 \%$ and the intensity of staining was moderate to strong. There was a clear tendency towards increasing the percentage of cells, expressing this marker, and the intensity of staining according to the group of benign diseases reaching highest values for the group of breast cancer. This shows that the markerYKL-40 can be used as a criterion for malignant degeneration, as well as for the differential diagnosis between different groups of benign breast diseases on one hand, and between benign diseases and breast cancer on the other.

In our study we compared the serum level of YKL-40 to its tissue expression. There was a real correlation between them. In nonproliferating diseases the serum level was near that of healthy women and the tissue expression was low and of weak intensity. In proliferating diseases without atypia the serum level of YKL-40 was higher than that of the first group, and the percentage of cells, expressing it was high and of moderate staining intensity. In proliferating diseases with atypia the serum level was significantly higher, reaching that of breast cancer, and the tissue expression was of moderate intensity with percentages of expressing cells between $50-75 \%$. In the group of breast cancer the serum level was 117,1 ; tissue expression was of high intensity and the percentage of expressing cells was $75-100 \%$. It is indicative that both the serum level of YKL-40 and its tissue expression in breast cancer are significantly higher than those in benign breast diseases - nonproliferating and proliferating ones without atypia. The differences in its concentration in proliferating diseases with atypia and breast cancer are not that significant. This may serve as an important criterion for the possible malignant transformation of an otherwise benign breast disease, as well as for the differential diagnosis between benign diseases and breast cancer.

\section{CONCLUSIONS}

For the first time in Bulgaria we examine the serum levels of YKL-40 in different groups of benign breast diseases, in breast cancer and in healthy controls. We draw a parallel between its serum concentration and tissue expression in the different groups. Thus we have the possibility to apply YKL-40 as a biomarker in the early diagnosis and differential diagnosis of benign and malignant breast diseases and the early detection of breast cancer.

\section{REFERENCES:}

1. Cintin C, Johansen JS, Christensen IJ, et al.Serum YKL-40 and colorectal cancer.Br J Cancer 1999, 79: 1494-1499.

2. Dupont J, Tanvar MK, Thaler HT,et 1.Early detection and prognosis of ovarian cancer using serum YKL-40 .J Clin Oncol 2004, 22:3330-3339.

3. Haagensen C.Diseases of the breast. Philadelphia: WB Saunders Company; 1986.

4. Johansen JS, Christensen IJ, Riisbo $r$ et al. High serum YKL-40 levels in patients with primary breast cancer is related to short reccurence free survival. Breast Cancer Res Treat 2003; 80: 15-21.

5. Johansen JS, Christensen IJ, Riisbro R, Greenall M, Han C, Price PA, Smith K, Brunner N, Harris Al: High serum YKL40 levels in patients vith primary breast cancer is related to short recurrence free survival.Btreast Cancer Res Treat 2003, $80: 15-21$.

6. Johansen JS, Drivsholm L, Price PA, et al.High serum YKL-40 level in patients vith small cell lung cancer is related to early death.Lung Cancer 2004; 46: 333-40.

7. Johansen JS. Studies on serum YKL40 as a biomarker in diseases with inflammation, tissue remodeling, fibrosis and cancer.Dan Med Bull, 2006;53:172209.

8. Johansen JS,Jensen BV, Roslind A, et al.Serum YKL-40, a new prognostic biomarker in cancer patients? Cancer Epidemiol Biomarkers Prev. 2006;15: 194202.

9. Johansen JS.Christensen IJ, Riisbro $\mathrm{R}$ et al.High serum YKL-40 levels in patients with primary breast cancer is related to short recurrence free survival. Breast Cancer Res Treat 203,80: 15-21.

10. Kim S., et al. Prognostic implications of immunohistochemically detected YKL-40 expression in breast cancer. Word Journal of Surgical Oncology 2007, 5: 1477-79.
11. Kim Sh, Das K, Shahla Norren S, et al. Prognostic implications of immunohistochemically detected YKL-40 expression in breast cancer.Word J Surg Oncol 2007; 5: 1-7.

12. Morrison BW, Leder P. Neu and ras initiate murine mammary tumors that share genetic markers generally absent in c- myc and int-2-initiated tumors. Oncogene 1994;9:3417-26.

13. Roslind A, et al. YKL-40 Expression in Benign and Malignant lesions of the Breast: A Methodologic Study. Appl Immunohistochem Mol Morphol 2007; 15: 371-381

14. Roslind A, Johansen JS,Junkern et al. YKL-40 expression in breast cancer.J Clin Oncol 2005, 23: 9665.

15. Valagussa P.Her- 2 status: a statistic wiew. Ann Oncol 2001,12:s29S34.

\author{
Address for correspondence: \\ Dr Victoria Sarafian \\ Department of Biology, Medical University - Plovdiv \\ 15a, Vasil Aprilov Blvd., 4000 Plovdiv, Bulgaria \\ Tel. +359 32/602 531; 265 986; \\ E-mail: sarafian@abv.bg
}

